\title{
sciendo
}

\author{
Current Issues in Pharmacy and Medical Sciences
}

Formerly ANNALES UNIVERSITATIS MARIAE CURIE-SKLODOWSKA, SECTIO DDD, PHARMACIA

journal homepage: http://www.curipms.umlub.pl/

\section{Scutellaria baicalensis - a small plant with large pro-health biological activities}

\author{
Karolina Wojtunik-Kulesza $^{1 \star}{ }^{\circledR}$, Kamila Kasprzak-Drozd ${ }^{1}{ }^{\circledR}$, Daniel Sajdlowski $^{2}$, \\ Anna OniszczuK ${ }^{1} \oplus$, Wojciech Swiatkowski ${ }^{2}$, Monika WaksmundzKa-Hajnos ${ }^{1}$
}

${ }^{1}$ Department of Inorganic Chemistry, Medical University of Lublin, Poland

${ }^{2}$ Chair and Department of Oral Surgery, Medical University of Lublin, Poland

\section{ARTICLE INFO}

Received 08 October 2020

Accepted 04 January 2021

\section{Keywords:}

Scutellaria baicalensis,

antioxidant,

Huang-Qin,

periodontal,

cardiovascular system.

\begin{abstract}
Scutellaria baicalensis, known also as Huang-Qin is a traditional Chinese plant used in medicine for at least 2000 years. The plant is widely distributed in Japan, Korea, Mongolia and Russia, and is listed in Chinese Pharmacopoeia, European Pharmacopoeia and British Pharmacopoeia. The interest in Huang-Qin results from various biological activities which are primarily related to secondary plants metabolites consisting of flavonoids, phenolic compounds and terpenes. It is known that the compounds are active against numerous diseases and protect the organism against harmful pathogenic agents. Particular attention is paid to baicalein, wogonin and oroxylin A - which are characteristic secondary metabolites of the plant. In this paper, we focused on phytochemical analysis and selected biological activities used in periodontal and cardiovascular problems. The presented studies confirm the ability of Huang-Qin to scavenge free radicals, moreover, that it presents anti-bacterial, anti-inflammatory and enzyme inhibitory activities.
\end{abstract}

\section{INTRODUCTION}

Herbal medicine is the raw or extracted products isolated from natural substances that have medicinal or life enhancing properties. In most cases, the substances are safer and less toxic than synthesized drugs and their pro-health properties significantly exceed side effects [1]. Today, scientists are focused on searching substances have effectiveness against cancer, neurodegeneration, viruses, especially SARS-CoV-2 and a lot of more. Nevertheless, such activity demands long and hard work involving a lot of people around the World.

Among the intensively studied plants is Scutellaria, a wide-spread genus commonly used in traditional Asiatic medicine. Scutellaria baicalensis belongs to the Lamiaceae family. It is a flowering plant with bitter taste and cold nature. The roots of the plant are widely used in traditional Chinese medicine, especially against viruses, microbials and inflammation $[2,3]$. Detail studies have revealed diverse biological activities which result from its rich composition of secondary plant metabolites (terpenes and flavonoids) [4]. In this paper, particular attention is paid to baicalin $\left(\mathrm{C}_{21} \mathrm{H}_{18} \mathrm{O}_{11}\right.$; 7 -D-glucuronic acid-5,6-dihydroxyflavone) derived from the roots of Scutellaria baicalensis Georgi. Detail analysis of the compound has indicated that it holds

\footnotetext{
* Corresponding author

e-mail: karolina.wojtunik@umlub.pl
}

antiviral, anti-tumor, anti-apoptotic, antioxidative, neuronal protective and anti-thrombotic properties [5-7]. Furthermore, recent, in-depth studies have demonstrated the significant biological activity of the plant, especially from derivatives from its stems and leaves, towards the protection of hippocampal neurons, which result in improvement in learning and memory, as well as reduction of oxidative damage [8].

This paper outlines the phytochemistry and biological activity of Scutellaria baicalensis.

\section{Phytochemistry}

Scutellaria baicalensis is a plant rich in secondary plant metabolites. These can be classified into four types: free flavonoids, flavonoid glycosides, phenylethanoid glycosides and other small molecules such as steroids, diterpenes, amides and phenolic compounds. Polysaccharides are an additional group that can be find in the plant [9]. It is known that the main source of active compounds is found in the plant's root, whereas its aerial parts are less affluent [3].

Among the aforementioned flavonoids, which include flavones, flavonols, flavonones, flavonol, chalcone and biflavonoid, the most intensively studied are baicalein, wogonin and oroxylin A (Fig. 1) of these, baicalin, a flavonoid glycoside, is of the greatest abundance, and was the first pure compound reported from the plant. An equally interesting 
secondary metabolite is wogonin - which was the first freeflavonoid isolated from Scutellaria baicalensis [3]<smiles>O=c1cc(-c2ccccc2)oc2cc(O)c(O)c(O)c12</smiles>

(1)

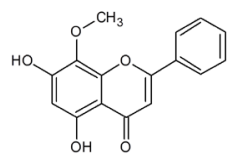

(2)

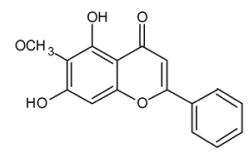

(3)
Figure 1. Structures of baicalein (1), wogonin (2) and oroxylin A (3), the most intensively studied secondary plant metabolites of Scutellaria baicalensis.

Table 1. Selected compounds identified in root and aerial parts of Scutellaria baicalensis

\begin{tabular}{|c|c|c|}
\hline Name & Plant part & References \\
\hline \multicolumn{3}{|l|}{ Free flavonoids } \\
\hline baicalein & $\begin{array}{c}\text { root, hairy } \\
\text { root }\end{array}$ & {$[10],[11]$} \\
\hline Scutellarein & root & {$[10]$} \\
\hline Oroxylin A & root & [11] \\
\hline Tenaxin II (5,7,2'-Trihydroxy-6-methoxyflavone) & root & [12] \\
\hline $5,7,4^{\prime}$-Trihydroxy-6-methoxyflavone & aerial part & [9] \\
\hline 5,7-Dihydroxy-6,8-dimethoxyflavone & root & [13] \\
\hline Chrysin (5,7-Dihydroxyflavone & root & [14] \\
\hline Norwogonin (5,7,8-Trihydroxyflavone) & root & [12] \\
\hline Apigenin $\left(5,7,4^{\prime}\right.$-Trihydroxyflavone) & root & [13] \\
\hline Wogonin (5,7-Dihydroxy-8-methoxyflavone) & root & [9] \\
\hline Scutevulin (5,7,2'-Trihydroxy-8-methoxyflavone) & root & {$[15]$} \\
\hline 5,7-Dihydroxy-8,2', 3',6'-tetramethoxyflavone & root & {$[16]$} \\
\hline Genkwanin (5,4'-Dihydroxy-7-methoxyflavone) & aerial part & [17] \\
\hline \multicolumn{3}{|l|}{ Flavonoid glycosides } \\
\hline Apigenin 7-O- $\beta$-D-glucoside & aerial part & [9] \\
\hline Baicalein 7-O- $\beta$-D-glucoside & root & {$[15]$} \\
\hline Oroxylin A 7-O- $\beta$-D-glucoside & aerial part & [9] \\
\hline $\begin{array}{l}5,6 \text { '-Dihydroxy-7,8-dimethoxyflavone } 2^{\prime} \text {-O- } \beta \text {-D- } \\
\text { glucoside }\end{array}$ & root & {$[18]$} \\
\hline Wogonin $5-O-\beta-D$-glucoside & root & [19] \\
\hline $3,5,7,6^{\prime}$-Tetrahydroxyflavone 2'-O- $\beta$-D-glucoside & root & {$[18]$} \\
\hline Kaempferol 3-O- $\beta$-D-glucoside & aerial part & {$[20]$} \\
\hline Wogonoside (Wogonin 7-O- $\beta$-D-glucuronide) & $\begin{array}{c}\text { root, hairy } \\
\text { root }\end{array}$ & [21] \\
\hline Apigenin 7-O- $\beta$-D-glucuronide & aerial part & {$[20]$} \\
\hline Chrysin 8-C- $\beta-D-$ glucoside & root & [18] \\
\hline \multicolumn{3}{|l|}{ Phenylethanoid glycosides } \\
\hline Darendoside B & root & {$[18]$} \\
\hline Acteoside & hairy root & {$[22]$} \\
\hline Isomartynoside & root & {$[23]$} \\
\hline Leucosceptoside A & hairy root & {$[23]$} \\
\hline Cistanoside D & root & [24] \\
\hline \multicolumn{3}{|l|}{ Others } \\
\hline Stigmasterol & root & [13] \\
\hline$\beta$-Sitosterol & root & {$[25]$} \\
\hline Pellitorine & root & [26] \\
\hline Benzoic acid & root & {$[25]$} \\
\hline Vanillin & root & [27] \\
\hline
\end{tabular}

\section{Antioxidant activity}

The most characteristic compounds of Scutellaria baicalensis are baicalein, baicalin and scutellarin. These are responsible for a variety of biological-medicinal activities, including antioxidant and free radical scavenging. Studies demonstrating the effectiveness of these compounds on free radicals are listed in Table 2 .

Table 2. Antioxidant activity of baicalein, baicalin and scutellarin

\begin{tabular}{|c|c|c|}
\hline Compound & Studies confirming antioxidant activities & References \\
\hline \multirow{4}{*}{ baicalein } & $\begin{array}{l}\text { Prevention of diabetes-related periodontal tissue } \\
\text { destruction by regulation of the Nrf2 signaling } \\
\text { pathway }\end{array}$ & [28] \\
\hline & $\begin{array}{l}\text { Determination of antioxidant activity of baicalein- } \\
p \text {-sulfonatoacalix } \mathrm{n} \text { ]arenes inclusion complexes }\end{array}$ & [29] \\
\hline & 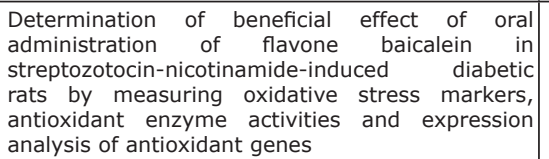 & [30] \\
\hline & $\begin{array}{l}\text { Protective mechanism of the antioxidant baicalein } \\
\text { toward hydroxyl radical-treated bone marrow- } \\
\text { derived mesenchymal stem cells }\end{array}$ & [31] \\
\hline \multirow{3}{*}{ baicalin } & $\begin{array}{l}\text { Studies towards baicalin suppression of apoptosis, } \\
\text { reactive oxygen species (ROS) generation, } \\
\text { NO production, foam cell formation, protein } \\
\text { expression of inducible nitric oxide synthase and } \\
\text { cyclooxygenase-2 in MØ-MP-induced RAW264 }\end{array}$ & [32] \\
\hline & $\begin{array}{l}\text { Enhancement of antioxidant activity of the inclusion } \\
\text { complexes of baicalin with p-sulfonatocalix[n] } \\
\text { arenes }\end{array}$ & [33] \\
\hline & $\begin{array}{l}\text { Antioxidant activity against superoxide and peroxyl } \\
\text { radical of baicalin and its oxidovanadium(IV) } \\
\text { complex }\end{array}$ & [34] \\
\hline \multirow{3}{*}{ scutellarin } & $\begin{array}{l}\text { Dual effect of glucuronidation of a pyrogallol-type } \\
\text { phytophenol antioxidant }\end{array}$ & [35] \\
\hline & $\begin{array}{l}\text { Investigation of the effects of Sc on IR and } \\
\text { oxidative stress and explored the underlying } \\
\text { mechanisms of action in vivo and vitro }\end{array}$ & [36] \\
\hline & $\begin{array}{l}\text { Examination of the effects of scutellarin on } \\
\text { oxidative stress-induced vascular endothelial } \\
\text { dysfunction and endothelial cell damage }\end{array}$ & [37] \\
\hline
\end{tabular}

The harmful activity of free radicals leads to numerous disorders such as cancer, neurodegeneration or tissue damage. One of several possible antioxidant mechanisms of Scutellaria baicalensis-based medicinals is based on NO production-inhibition. Studies performed towards the activity of compounds isolated from $\mathrm{MeOH}$ extracts of Scutellaria baicalensis have revealed that three contained show inhibition levels above $83 \%$ [38]. Another study, based on EtOH extract of the plant confirmed the contained antioxidant activity by demonstrating its ability to scavenge DPPH. The obtained results were correlated with the amount of total polyphenol content, and this correlation demonstrated significant values in the relationship between the investigated phenolic compounds and the reducing power of the plant's extracts [39]. Similar activity was observed for baicalein, baicalin and scutellarin when analyzed via DPPH, ABTS and CAA assays. The three flavonoids made contributions of $58.33 \%, 60.36 \%$ and $51.41 \%$ (respectively) to the overall antioxidant activity of a flavonoid-rich extract [40]. Of note, a study of the comparison of the antioxidant activity of extracts obtained from root, leaf, stem and flower performed by Seo et al. revealed high antioxidant activity for all used methods (DPPH, ABTS and RP), nevertheless, the highest activity was observed for root, followed by leaf, stem and flower [41]. 
Beside in vitro studies, antioxidant activity was also evaluated in in vivo conditions. For example, Peng et al. [42] performed an assay based on mice bearing U14 cervical cancers. Here, the researchers observed an increase in activity of SOD and a decrease in MDA level. This outcome demonstrated the significant antioxidant activity of $S$. baicalensis extract and revealed that the plant's extracts can be considered as potential inhibitors of tumor growth. Confirmation of the effect resulted from studies performed with use of D-galactose and $\mathrm{NaNO}_{2}$-injected mice. In this case, high dose of extract (100 mg/ $\mathrm{kg}$ ) showed significant influence on SOD, catalase activities and MDA levels [43].

\section{Influence on periodontal tissues}

Due to its biological activities, derivatives of S. baicalensis have been investigated for their usefulness in various branches of pharmacy and medicine. For example, in dentistry, extracts of the plant are applicable due to their high antioxidant, antibacterial, protective and anti-inflammatory activities. A significant source of periodontal problems is the oral bacteria that is present in dental plaque. The pathogenic bacteria activate immunological cells that produce various mediators and effectors of tissues breakdown [44]. One possible way to counteract the problem is antibacterial therapy. Currently, numerous toothpastes and mouth rinse are rich in natural herbal ingredients. Among these are derivatives of $S$. baicalensis, as these have revealed anti-bacterial effect on oral pathogens. The studies performed by Leung et al., for example, aimed at encapsulating $S$. baicalensis with chlorhexidine. The resulting nanoparticles revealed a synergistic effect against mixed oral bacterial biofilms [45].

Baicalin, one of the secondary metabolite of $S$. baicalensi, has important influences upon periodontal tissues, and numerous animal studies have shown the positive impact of the compound on periodontitis. The periodontal ligament cells (PDLC) are the main cells of the periodontal ligament. Cao et al. [46] focused on type I collagen and the effect of baicalin upon these, particularly, the expression of type I collagen mRNA in PDLC. They noted a positive effect. In Li et al. [47] investigation of the activity of baicalin towards periodontitis lesions, baicalin turned out to be active towards reducing the number of osteoclasts increased by LPS. The positive influence of baicalin was also recorded in the synthesis of collagen, while baicalin exhibited a prominent impact on the process, as well as on the synthesis of total protein from human gingival fibroblasts. This activity is connected with the inhibitory impact of the secondary metabolites on collagenase - which is able to destroy collagen tissue [48].

Additionally, the positive effect of baicalin application was observed towards pro-inflammatory mediators. It is known that one of possible way of treating chronic inflammation is down-regulation of over-expressed TNF- $\alpha$ and IL-1 $\beta$, both of which are responsible for activating NF-кB [49]. Another important enzyme linked with inflammation is cyclooxygenase-2 (COX-2). In the case of periodontal disease, the problem could be resolved by inhibiting the enzyme. The issue was considered by Cai et al. [50], whose studies revealed the positive influence of baicalin on upregulation of $\mathrm{COX}-2$ protein expression in macrophages, plasma cells, fibroblasts and gingival tissues in an animal model of ligature-induced periodontitis. Considering periodontal disease, attention should be also paid to cyclooxygenase, nitrogen metabolites, reactive oxygen and cytokines. Here, by means of baicalin application, iNOS, an inducible nitric oxide synthase responsible for overproduction of nitric oxide in periodontitis, inhibition was observed [50].

\section{Cardiovascular and cerebrovascular diseases}

It has been demonstrated that the Scutellaria baicalensis can influence the course of cardiovascular and cerebrovascular diseases [3]. Factors involved in the pathogenesis of many cardiovascular diseases include abnormal mitochondrial fission and mitophagy. Baicalein inhibits mitochondrial apoptosis induced by oxidative stress in cardiomyocytes by stabilizing MARCH5 expression. In addition, it may counteract cardiotoxicity. One of the mechanisms of such action is the inhibition of mitochondrial apoptosis induced by oxidative stress in cardiomyocytes by stabilizing MARCH5 expression. Overexpressed MARCH5 reduces apoptosis of myocardial cells by countervailing oxidative stress as well as ischaemia and reperfusion [51].

Source data indicate that baicalein has antiatherosclerotic properties, such as macrophage lipid regulation [52]. Accordingly, Scutellaria baicalensis Georgi extract (containing baicalin, baicalein and wogonin) has demonstrated antioxidant and anti-inflammatory properties against macrophages. It has also been shown that it inhibited the $\mathrm{Cu}^{2+}$ - induced oxidation of LDL, the expression of NO generation and inducible NO synthase, and exhibited an antiinflammatory property partly via the induction of $\mathrm{HO}^{-1}$ [53].

Tissue factor (TF) is the most common cause of arteriosclerotic thrombosis, and plaque atherosclerotic rupture, followed by thrombosis. TF can cause a heart attack or stroke. Wogonin is a biologically active compound that is active in countering the effects of inflammatory diseases, atherosclerosis and hyperlipidemia. Wogonin is known to affect TF expression. In addition, Wogonin acts as an anticoagulant on the basis of various mechanisms of action. For example, it dose dependently inhibits mRNA, protein and PMA-enhanced TF activity at EC. This inhibition was attributed to the decreasing nuclear accumulation of transcription factors phospho-c-Jun and early growth-1 (Egr-1) responses. Furthermore, it showed anticoagulant activity by inhibiting TF expression and activity in tumor necrosis factor-alpha (TNF- $\alpha$ ) - and lipopolysaccharide (LPS) -treated ECs and THP-1 cells. It is also possible that vogonin limits the transactivation of TF promoter activity, leading to regulation of TF expression and activity induced by inflammatory mediators [54].

Diabetes mellitus initiates many metabolic disorders in various tissues and organs, including the cardiovascular system. Such disorders can lead to heart failure, even in the absence of ischemic heart disease. This is known as 'diabetic cardiomyopathy'. Research on the effectiveness of wogonin in controlling diabetic cardiomyopathy is ongoing [55].

Wang et al. [56] investigated the effect of baicalin on hyperglycemia-induced cardiovascular malformations during embryonic development. For this purpose, they used early chicken embryos - they estimated the optimal 
concentration of baicalin for preventing these defects as $6 \mu \mathrm{M}$. This research indicated that Baicalin reduced hyperglycemia-induced cell apoptosis, inhibited excessive ROS (reactive oxygen species) production, as well as regulated SOD (superoxide dismutase), d GSH-Px (glutathione peroxidase) and GABAA (c-aminobutyric acid) levels [56].

Inflammatory processes are an important aspect in the pathogenesis of different heart diseases such as myocardial fibrosis, atherosclerosis and myocardial depression. BNP (brain natriuretic peptide) level is considered as a diagnostic marker of hypertropy and heart failure. An experiment in an animal model with spontaneously hypertension (rats) has shown that baicalein $(200 \mathrm{mg} / \mathrm{kg} / \mathrm{day}$, intraperitoneally) protects myocardial fibrosis by significantly reducing collagen deposition in left ventricular tissue. Moreover, its administration alleviated elevated systolic blood pressure and BNP levels in plasma by suppressing the expression of 12-LOX, p-ERK and MMP-9 in heart tissue. In addition, there was a decrease in the thickness of the interventricular septum and the body weight/heart ratio. The anti-fibrotic effect of baicalein in the experiment was compared to that of valsartan at a dose of $20 \mathrm{mg} / \mathrm{kg} /$ day [57].

\section{CONCLUSION}

S. baicalensis is a well-known and commonly used plant in Chinese traditional medicine. The various recognized biological activities have encourage the dissemination of the plant into European medicine and pharmacy. Phytochemical analysis has revealed that it contains various secondary plant metabolites that are active against numerous factors leading to diseases such as neurodegenerations, cardiovascular and cerebrovascular or periodontal problems. We can, therefore, observe an increase in interest in the potential use of $S$. baicalensis, as well as its inclusion in more and more natural medicaments and cosmetics. What is more, the multidirectional activity of Huang-Qin has given hope that it can be active against neurodegeneration or cancer.

\section{FINANCIAL SUPPORT AND SPONSORSHIP}

Nil.

\section{CONFLICTS OF INTEREST}

Authors of this work have no conflicts of interest.

\section{ORCID iDs}

\section{Karolina Wojtunik-Kulesza}

(D)https://orcid.org/0000-0002-6541-8817 Kamila Kasprzak-Drozd (1)https://orcid.org/0000-0002-6282-6313 Anna Oniszczuk (Dhttps://orcid.org/0000-0002-5109-3302

\section{REFERNCES}

1. Brezniak N, Wasserstein A. Orthodontically induced inflammatory root resorption. Part I: The basic science aspects. Angle Orthod. 2002; 72:175-9.

2. Suh MG, Choi HS, Cho K, Park SS, Kim WJ, Suh HJ, et al. Antiinflammatory action of herbal medicine comprised of Scutellaria baicalensis and Chrysanthemum morifolium. Biosci Biotechnol Biochem. 2020;84:1799-09.
3. Xia YT, Chana GKL, Wanga HY, Donga TTX, Duan R, Hua WH, et al. The anti-bacterial effects of aerial parts of Scutellaria baicalensis: Potential application as an additive in aquaculture feedings. Aquaculture. 2020;526:735418.

4. Wang ZL, Wang S, Kuang Y, Hu ZM, Qiao X, Ye M. A comprehensive review on phytochemistry, pharmacology, and flavonoid biosynthesis of Scutellaria baicalensis. Pharm Biol. 2018;56:465-86.

5. Gong GW, Wang HY, Kong XP, Duan R, Dong TX, Tsim WK. Flavonoids are identified from the extract of Scutellariae Radix to suppress inflammatory-induced angiogenic responses in cultured RAW 264.7 macrophages. Sci Report. 2018;8:17412.

6. Chan FL, Choi HL, Chen ZY, Chan PS, Huang Y. Induction of apoptosis in prostate cancer cell lines by a flavonoid, baicalin. Cancer Lett. 2000;160:219-28.

7. Cheng F, Lu Y, Zhong X, Song W, Wang X, Sun X, Qin J, et al. Baicalin's therapeutic time window of neuroprotection during transient focal cerebral ischemia and its antioxidative effects in vitro and in vivo. Evid Complement Alternat Med. 2013, 120261.

8. Cheng CS, Chen J, Tan HY, Wang N, Chen Z, et al. Scutellaria baicalensis and Cancer treatment: recent progress and perspectives in biomedical and clinical studies. Am J Chin Med. 2018;46:25-54.

9. Song J, Zhou YZ, Panga YY, Gao L, Du GH, Qin XM. The anti-aging effect of Scutellaria baicalensis Georgi flowers extract by regulating the glutamine-glutamate metabolic pathway in D-galactose induced aging rats. Rev Aquac. 2020;134:110843.

10. Ma JL. Study on chemical constituents from stems and leaves of Scutellaria baicalensis. Chin J Exp Trad Med Formulae. 2013;19:147-9.

11. Popova TP, Litvinenko VI, Kovalev IP. Flavones of the roots of Scutellaria baicalensis. Chem Nat Compound. 1973;9:699-702.

12. Ji S, Li ZW, Song W, Wang YR, Liang WF, Li K, et al. Bioactive constituents of Glycyrrhiza uralensis (licorice): Discovery of the effective components of a traditional herbal medicine. J Nat Product. 2016;79:281-92.

13. Tomimori T, Miyaichi Y, Imoto Y, Kizu HA, Tanabe Y. Studies on the constituents of Scutellaria species II. On the flavonoid constituents of the roots of Scutellaria baicalensis Georgi. Yakugaku Zasshi. 1983;103:607-11.

14. Wang HY. Studies on anxiolytic constituents of Scutellaria baicalensis Georgi [dissertation]. Liaoning (Shenyang): Shenyang Pharmaceutical University. 2002. Chinese.

15. Takagi S, Yamaki M, Inoue K. Studies on the water-soluble constituents of the roots of Scutellaria baicalensis Georgi (wogon). Yakugaku Zasshi. 1980;100:1220-4.

16. Tomimori T, Miyaichi Y, Imoto Y, Kizu H, Suzuki C. Studies on the constituents of Scutellaria species. IV. On the flavonoid constituents of the root of Scutellaria baicalensis Georgi. Yakugaku Zasshi. 1984;104:529-34.

17. Long HL, Xu GY, Deng AJ, Li ZH, Ma L, Lu Y, et al. Two new flavonoids from the roots of Scutellaria baicalensis. J Asian Nat Product Res. 2015;17:756-60.

18. Wang HW, Yin ZF, Li HB, Yuan XX, Yang MM, Zhao GQ. Chemical constituents from stems and leaves of Scutellaria baicalensis. Chin J Exp Trad Med Formulae. 2016;22:41-4.

19. Miyaichi Y, Tomimori T. Studies on the constituents of Scutellaria Species XVII: phenol glycosides of the root of Scutellaria baicalensis Georgi (2). J Nat Med. 1995;49:350-3.

20. Takagi S, Yamaki M, Inoue K. Flavone di-C-glycosides from Scutellaria baicalensis. Phytochemistry. 1981;20:2443-44.

21. Cha JH, Kim HW, Kim S, Jung SH, Whang WK. Antioxidant and antiallergic activity of compounds from the aerial parts of Scutellaria baicalensis Georgi. Yakhak Hoeji. 2006;50:136-43.

22. Ishimaru K, Nishikawa K, Omoto T, Asai I, Yoshihira K, Shimomura K. Two flavone 2'-glucosides from Scutellaria baicalensis. Phytochemistry. 1995;40:279-81.

23. Zhou Y, Hirotani M, Yoshikawa T, Furuya T. Flavonoids and phenylethanoids from hairy root cultures of Scutellaria baicalensis. Phytochemistry. 1997;44:83-7.

24. Miyaichi Y, Tomimori T. Studies on the constituents of Scutellaria species XVI phenol glycosides of the root of Scutellaria baicaleinsis Georgi. J Nat Med. 1994;48:215-8. 
25. Ji S, Li R, Wang Q, Miao WJ, Li ZW, Si LL, Qiao X, Yu SW, Zhou DM, Ye M. Anti-H1N1 virus, cytotoxic and Nrf2 activation activities of chemical constituents from Scutellaria baicalensis. J Ethnopharmacol. 2015;176:475-84.

26. Xu DY, Chen PD, Zhang L, Cao YD, Ding AW. Study on chemical constituents of Scutellaria baicalensis. Chin J Exp Trad Med Formulae. 2011;17:78-80.

27. Xu JX, Ding LQ, Jiang MM, Qiu F. Non-flavonoid constituents from the roots of Scutellaria baicalensis Georgi. Chin J Med Chem. 2016;26:480-3.

28. Liu YX, Liu ZG, Su L, Yang RP, Hao DF, Pei YH. Chemical constituents from Scutellaria baicalensis Georgi. Chin J Med Chem. 2009;19:59-62.

29. Zhu C, Zhao Y, Wu X, Qiang C, Liu J, Shi J, Gou J, et al. The therapeutic role of baicalein in combating experimental periodontitis with diabetes via Nrf2 antioxidant signaling pathway. J Periodont Res. 2020;55:381-91.

30. Chao J, Xu M, Wang X, Guo, Z. Baicalein-p-sulfonatocalix[n] arenes inclusion complexes: characterization, antioxidant ability and stability. Polym Bull. 2019;76:989-1006.

31. Sarkar P, Nath K, Banu S. Modulatory effect of baicalein on gene expression and activity of antioxidant enzymes in streptozotocinnicotinamide induced diabetic rats. Braz J Pharm Sci. 2019;55:e18201.

32. Tian Y, Li X, Xie H, Wang X, Xie Y, Chen C, Chen D. Protective mechanism of the antioxidant baicalein toward hydroxyl radicaltreated bone marrow-derived mesenchymal stem cells. Molecules. 2018;23:223.

33. Paudel KR, Kim D.W. Microparticles-mediated vascular inflammation and its amelioration by antioxidant activity of baicalin. Antioxidants. 2020;9:890.

34. Chao J, Wang X, Xu M, Zuo Y. Characterization and enhanced antioxidant activity of the inclusion complexes of baicalin with p-sulfonatocalix[n]arenes. J Incl Phenom Macrocycl Chem. 2019;93:361-70

35. Martínez Medina JJ, Nasob LG, Pérez AL, Rizzi A, Ferrer EG, Williams PAM. Antioxidant and anticancer effects and bioavailability studies of the flavonoid baicalin and its oxidovanadium (IV) complex. J Inorg Biochem. 2017;166:150-61.

36. Liu Q, Li X, Ouyang X, Chen D. Dual effect of glucuronidation of a pyrogallol-type phytophenol antioxidant: A comparison between scutellarein and scutellarin. Molecules. 2018;23:3225.

37. Gao L, Tang H, Zeng, Tang T, Chen M, Pu P. The anti-insulin resistance effect of scutellarin may be related to antioxidant stress and AMPKa activation in diabetic mice. Obes Res Clin Pract. 2020;14:368-74.

38. Mo J,Yang R, Li F, Zhang X, He B, Zhang Y, Chen P, Shen Z. Scutellarin protects against vascular endothelial dysfunction and prevents atherosclerosis via antioxidation. Phytomedicine. 2018;42:66-74.

39. Han YK, Kim H, Shin H, Song J, Lee MK, Park B, Lee KY. Characterization of anti-inflammatory and antioxidant constituents from Scutellaria baicalensis using LC-MS coupled with a bioassay method. Molecules. 2020;25:3617.

40. Vergun O, Svydenko L, Grygorieva O, Shymanska O, Rakhmetov D, Brindza J, Ivanišová E, Antioxidant capacity of plant raw material of Scutellaria baicalensis Georgi. Potr Slovac J Food Sci. 2019;13:614-21.

41. Li K, Fan H,Yin P, Yang L, Xue Q, Li X, et al. Structure-activity relationship of eight high content flavonoids analyzed with a preliminary assign-score method and their contribution to antioxidant ability of flavonoids-rich extract from Scutellaria baicalensis shoots. Arab J Chem. 2018;11:159-70.
42. Seo ON, Kim GS, Kim YH, Sung SP, Soo WJ, Jong JL, et al. Determination of polyphenol components of Korean Scutellaria baicalensis Georgi using liquid chromatography - tandem mass spectrometry: Contribution to overall antioxidant activity. J Funct Foods. 2013;5:1741-50.

43. Peng Y, Guo CS, Li PX, Fu ZZ, Gao LM, Di Y, et al. Immune and Anti-oxidant Functions of Ethanol Extracts of Scutellaria baicalensis Georgi in Mice Bearing U14 Cervical Cancers. Asian Pac J Cancer Prev. 2014;15:4129-33.

44. Jeong K, Shin YC, Park S, Park JS, Kim N, Um JY, et al. Ethanol extract of Scutellaria baicalensis Georgi prevents oxidative damage and neuroinflammation and memorial impairments in artificial senescense mice. J Biomed Sci. 2011;18:14.

45. Ming J, Zhuoneng L, Guangxun Z. Protective role of flavonoid baicalin from Scutellaria baicalensis in periodontal disease pathogenesis: A literature review. Complement Ther Med. 2018;38:11-8.

46. Leung KC, Seneviratne CJ, Li X, Leung PC, Lau CBS, Wong CH, et al. Synergistic antibacterial effects of nanoparticles encapsulated with Scutellaria baicalensis and pure chlorhexidine on oral bacterial biofilms. Nanomaterials (Basel). 2016;6:61.

47. Cao Z, Li C, Zhu G. Inhibitory effects of baicalin on IL-1beta-induced MMP-1/TIMP-1 and its stimulated effect on collagen-1 production in human periodontal ligament cells. Eur J Pharmacol. 2010;641:1-6.

48. Li H, Li G, Liu X, Qiao Y, Cui J, Liu Z. The histological observation of the inhibitory effects of baicalin in lipopolysaccharide-induced periodontitis in rats. J Pract Stomatol. 2016;32:133-4.

49. Chung CP, Park JB, Bae KH. Pharmacological effects of methanolic extract from the root of scutellaria baicalensis and its flavonoids on human gingival fibroblast. Planta Med. 1995;61:150-3

50. Doyle SL, O'Neill LA. Toll-like receptors: from the discovery of NF- $\kappa B$ to new insights into transcriptional regulations in innate immunity. Biochem Pharmacol. 2006;72:1102-13.

51. Cai X, Li C, Du G, Cao Z. Protective effects of baicalin on ligatureinduced periodontitis in rats. J Periodontal Res. 2008;43:14-21.

52. Li Q, Yu Z, Xiao D, Wang Y, Zhao L, An Y, et al. Baicalein inhibits mitochondrial apoptosis induced by oxidative stress in cardiomyocytes by stabilizing MARCH5 expression. J Cell Mol Med. 2020;24:2040-51.

53. Jian X, Liu Y, Zhao Z, Zhao L, Wang D, Liu Q. The role of traditional Chinese medicine in the treatment of atherosclerosis through the regulation of macrophage activity. Biomed Pharmacother. 2019;118:109375.

54. Kim OS, Seo CS, Kim Y, Shin HK, Ha H. Extracts of Scutellariae Radix inhibit low-density lipoprotein oxidation and the lipopolysaccharide-induced macrophage inflammatory response. Mol Med Rep. 2015;12:1335-41.

55. Wu YH, Chuang LP, Yu CL, Wang SW, Chen HY, Chang YL. Anticoagulant effect of wogonin against tissue factor expression. Eur J Pharmacol. 2019;859:172517.

56. Khan S, Kamal M. Can wogonin be used in controlling diabetic cardiomyopathy? Curr Pharm Des. 2019;25:2171-7.

57. Wang G, Liang J, Gao LR, Si ZP, Zhang XT, Liang G. et al. Baicalin administration attenuates hyperglycemia-induced malformation of cardiovascular system. Cell Death Dis. 2018;9:1-17.

58. Dinda B, Dinda S, DasSharma S, Banik R, Chakraborty A, Dinda M. Therapeutic potentials of baicalin and its aglycone, baicalein against inflammatory disorders. Eur J Med Chem. 2017;131:68-80. 